\title{
Usefulness of Coronary Flow Reserve Measured by Echocardiography to Improve the Identification of Significant Left Anterior Descending Coronary Artery Stenosis Assessed by Multidetector Computed Tomography
}

\author{
Francesco Pizzuto, MD ${ }^{\mathrm{a}, *}$, Paolo Voci, MD ${ }^{\mathrm{a}}$, Francesco Bartolomucci, MD ${ }^{\mathrm{b}}$, \\ Paolo Emilio Puddu, MD ${ }^{\mathrm{c}}$, Giovanni Strippoli, $\mathrm{MD}^{\mathrm{b}}$, Laura Broglia, MD ${ }^{\mathrm{d}}$, and Plinio Rossi, $\mathrm{MD}^{\mathrm{e}}$
}

\begin{abstract}
Multidetector computed tomography (MDCT) detects coronary artery disease. However, an overestimation of coronary artery stenosis and artifacts can prevent accurate identification of significant coronary narrowing. The combination of MDCT with coronary flow reserve (CFR), the hyperemic/baseline peak flow velocity ratio, measured by transthoracic Doppler echocardiography might be helpful. We studied 144 consecutive patients with CFR and quantitative coronary angiography, obtained using both MDCT and invasive coronary angiography (reference method). It was hypothesized that the CFR might provide an incremental value to MDCT in detecting significant $(\geq 70 \%)$ left anterior descending (LAD) coronary artery stenosis. A CFR cutoff of $<2$ was used to discriminate significant stenosis. CFR was feasible in $141(98 \%)$ of 144 patients, and MDCT was feasible in 131 $(91 \%)$ of 144 patients $(\mathrm{p}<0.02)$. In a univariate model, the prediction of significant LAD stenosis was slightly, but significantly ( $\mathrm{c}<0.0001$ ), better with CFR (sensitivity $90 \%$, specificity $96 \%$, positive predictive value $84 \%$, negative predictive value $97 \%$, and diagnostic accuracy $94 \%$, chi-square $=97.5$ ) than with MDCT (sensitivity $80 \%$, specificity $93 \%$, positive predictive value $71 \%$, negative predictive value $95 \%$, diagnostic accuracy $90 \%$, chi-square $=63.2$ ). When the findings from transthoracic Doppler echocardiography and MDCT agreed, the diagnostic accuracy increased $(96 \%$; chi-square $=86.1, \mathrm{p}<0.0001)$. In a multivariate prediction of significant LAD stenosis using a logistic neural network, CFR overshadowed MDCT, and the area under the receiver operating curve was 0.99 . Of the 13 patients missed by MDCT, the diagnostic accuracy of transthoracic Doppler echocardiography to predict significant LAD stenosis was $100 \%$. Thus, CFR could improve the diagnostic accuracy of MDCT to detect significant LAD stenosis. (C) 2009 Elsevier Inc. All rights reserved. (Am J Cardiol 2009;104:657-664)
\end{abstract}

Noninvasive coronary angiography by multidetector computed tomography (MDCT) has been increasingly used as a diagnostic tool to study the coronary anatomy in patients with suspected coronary artery disease and/or multiple risk factors. ${ }^{1-6}$ Agreement in published studies is substantial that negative findings on MDCT virtually exclude coronary atherosclerosis; however, quantitation of coronary artery stenosis is difficult. ${ }^{1-6}$ Therefore, a noninvasive multimodality imaging approach integrating morphologic and functional information has been suggested. ${ }^{7}$ Transthoracic color Doppler echocardiography (TDE) allows the measure-

\footnotetext{
aDepartment of Cardiology, "Tor Vergata" University, Rome, Italy; bDepartment of Cardiology, Community Hospital "L. Bonomo," Andria (BA), Italy; 'Department of the Heart and Great Vessels "A. Reale," "La Sapienza" University, Rome, Italy; ${ }^{\mathrm{d}}$ Department of Radiology, "S. Giovanni-Addolorata" General Hospital, Rome, Italy; and "Department of Radiology, Clinica "Villa Margherita," Rome, Italy. Manuscript received February 5, 2009; revised manuscript received and accepted April 27, 2009.

*Corresponding author: Tel: (+39) 06-8621-0144; fax: (+39) 06-86210144.

E-mail address: fbpizzuto@gmail.com (F. Pizzuto).
}

ment of the coronary flow reserve (CFR) in the left anterior descending (LAD) coronary artery, ${ }^{8}$ a useful parameter to detect significant ( $\geq 70 \%$ ) narrowing in the native coronary artery, ${ }^{8}$ as well as in the stented and/or grafted LAD artery. $^{9-12}$ The CFR might provide incremental functional information on the collateral circulation when chronic total coronary occlusion is present. ${ }^{13}$ We compared the value of LAD CFR versus MDCT to predict significant ( $\geq 70 \%$ ) LAD stenosis, using invasive coronary artery angiography as the reference method.

\section{Methods}

A total of 144 consecutive patients (106 men, 38 women, mean age 59 years, range 28 to 84 ) with suspected or proven coronary artery disease (Table 1) underwent both invasive and noninvasive (MDCT) coronary angiography and functional assessment of LAD CFR by TDE. Functional Doppler studies were performed at the end of MDCT and within 48 hours of invasive coronary angiography. The exclusion criteria for the CFR test were acute coronary syndromes, previous LAD-related myocardial infarction, congestive heart failure, significant valvular heart disease, contraindications to adenosine administration. The exclusion criteria 
Table 1

Study population $(\mathrm{n}=144)$

\begin{tabular}{ll}
\hline Symptoms & Patients (n) \\
\hline $\begin{array}{l}\text { Suspected coronary artery disease } \\
\text { Typical chest pain without previous ischemic events }\end{array}$ & $34(24 \%)$ \\
$\quad$ Atypical chest pain & $25(17 \%)$ \\
Proven coronary artery disease & $20(14 \%)$ \\
$\quad$ Previous left internal mammary artery graft & $11(8 \%)$ \\
Previous left anterior descending coronary artery stent & \\
Previous myocardial infarction, non-left anterior & $28(19 \%)$ \\
$\quad$ descending coronary artery related & \\
Previous acute coronary syndrome & $26(18 \%)$
\end{tabular}

for MDCT were atrial fibrillation, frequent extrasystolic beats, renal failure, and pregnancy. Patients with previous LAD stenting $(\mathrm{n}=11)$ and a left internal mammary artery (LIMA) to the LAD graft $(n=20)$ were included. All patients were in sinus rhythm and fasting state during each evaluation and provided written informed consent.

All multidetector computed tomographic studies were performed using 40-channel scanners (Brilliance CT, Philips Medical Systems, Eindhoven, The Netherlands). Oral and/or intravenous metoprolol $(\mathrm{n}=125)$ or oral verapamil $(n=9)$ were administered to obtain a heart rate of $\leq 60$ beats/min in all patients. Dedicated software allowed correction for R-wave irregularity after data acquisition. Sublingual nitroglycerine $(5 \mathrm{mg})$ was administered 5 to 10 minutes before contrast injection. An intravenous 18-gauge cannula was inserted in an antecubital vein and connected to a dual-head power injector (Stellant D, Medrad, Pittsburgh, Pennsylvania). For multidetector computed tomographic angiography, 100 to $120 \mathrm{ml}$ of nonionic iodinated contrast material (Iomeron, $400 \mathrm{mg}$ iodine $/ \mathrm{ml}$, Bracco, Milano, Italy, or Omnipaque, $350 \mathrm{mg} / \mathrm{ml}$, GE Healthcare AS, Oslo, Norway) was injected at 4 to $5 \mathrm{ml} / \mathrm{s}$ and immediately followed by a $30 \mathrm{ml}$ saline flush. A $40 \times 0.625-\mathrm{mm}$ collimation was used for scan acquisition. Other scan parameters were adjusted according to the patient's body habitus (tube voltage 120 to $140 \mathrm{kVp}$, tube current 450 to $850 \mathrm{mAs}$ ). The spiral pitch (0.2 to 0.3$)$ was adjusted according to the patient's heart rate, resulting in an average temporal resolution of 125 to $210 \mathrm{~ms}$, using an adaptive multisegment reconstruction algorithm ${ }^{14}$ and a gantry rotation time of 0.42 second. In 52 patients with a stable heart rate $<60$ beats/min at rest and no arrhythmia during scan preparation, electrocardiographically based dose modulation was implemented at a singlephase or dual phases according to the patient's heart rate. Image acquisition was automatically initiated using bolus tracking in the region of interest in the descending aorta, and the scan was performed during a single 14-second breathhold time. The electrocardiographic track was acquired during the scan, and image reconstruction was performed with retrospective gating by selecting the motionless data set throughout the RR interval. Images were reconstructed at a thickness of $0.64 \mathrm{~mm}$ and a 180 to $220-\mathrm{mm}$ field of view at several predetermined consistent physiologic phases of the cardiac cycle according to the heart rate. ${ }^{14,15}$ The reconstructed axial slices were stacked in a volume to generate a 3-dimensional data set. In patients with a LAD stent, not only the stented tract, but also the entire coronary vessel was evaluated. A stent was considered occluded when its lumen was darker than the contrast-enhanced vessel before the stent and/or when no distal runoff occurred. Significant in-stent restenosis was considered when the lumen inside the stent showed a darker rim (eccentric or concentric) between the stent and the enhanced vessel lumen, with a lumen reduction of $\geq 70 \%$. A larger volume of contrast was needed in patients with coronary artery bypass grafting to cover by the scan volume the origin and very proximal part of the LIMA. After a visual classification of the LIMA-todistal LAD artery segment as either "evaluable" or "unevaluable," the conduits were assessed for the presence of occlusion or significant stenosis. The proximal LAD artery was neglected in the evaluation, because this segment is functionally excluded by the graft, and the amount of calcium often prevents a clear imaging. Overall, the scan time (including the breath hold) was usually $<20$ seconds in these patients, depending on the additional time needed to image the distal LAD beyond the LIMA. The multidetector computed tomographic data sets were analyzed independently and on site by a cardiologist and radiologist, who were unaware of the clinical findings, and were always performed before the functional Doppler studies and invasive angiography. Differences in interpretation were resolved by consensus or by a binding third opinion. Postprocessing and data analysis took longer than the multidetector computed tomographic examination (30 to 45 minutes in most cases). Multiple image sets corresponding to different cardiac phases were simultaneously examined in a dedicated workstation (Brilliance Workspace, Philips Medical Systems), and the cardiac phases with the best segmentspecific image quality were selected for analysis. Multiplanar reformatting, curved multiplanar reformatting, maximum intensity projection, and volume rendering technique images were obtained for each coronary segment. For comparison with invasive quantitative coronary angiography, the degree of diameter stenosis (maximum diameter reduction) was determined in longitudinal curved multiplanar reformatted reconstructions by dividing the minimal diameter in the diseased segment by the diameter in the adjacent proximal disease-free section in the same projection used for invasive quantitative coronary angiography. Segments were considered unsuitable for interpretation if they had poor contrast opacification or artifacts caused by motion, ectopic beats, and/or calcium blooming. The total time for MDCT was typically 10 to 15 minutes.

The LAD was imaged by transthoracic Doppler ultrasonography, as previously described, ${ }^{9,11,12}$ in the left lateral decubitus, using a small multi-Hertz transducer connected to an ultrasound system (Sequoia C256, Siemens-Acuson, Mountain View, California), allowing independent changes of frequency between two-dimensional (3.5 to $7.0 \mathrm{MHz}$ ) and color Doppler ultrasonography (3.5 to $6.0 \mathrm{MHz}$ ). The LAD flow velocities were measured using pulsed Doppler ultrasonography with a color-coding guide. In brief, the distal LAD was imaged from the fourth to the fifth left intercostal space in the interventricular groove, using a modified 2-chamber view. ${ }^{9}$ Care was taken to visualize the most distal tract of the artery in the periapical area. The best long-axis view in color Doppler flow imaging was obtained to maintain a $<30^{\circ}$ angle between the flow and Doppler 

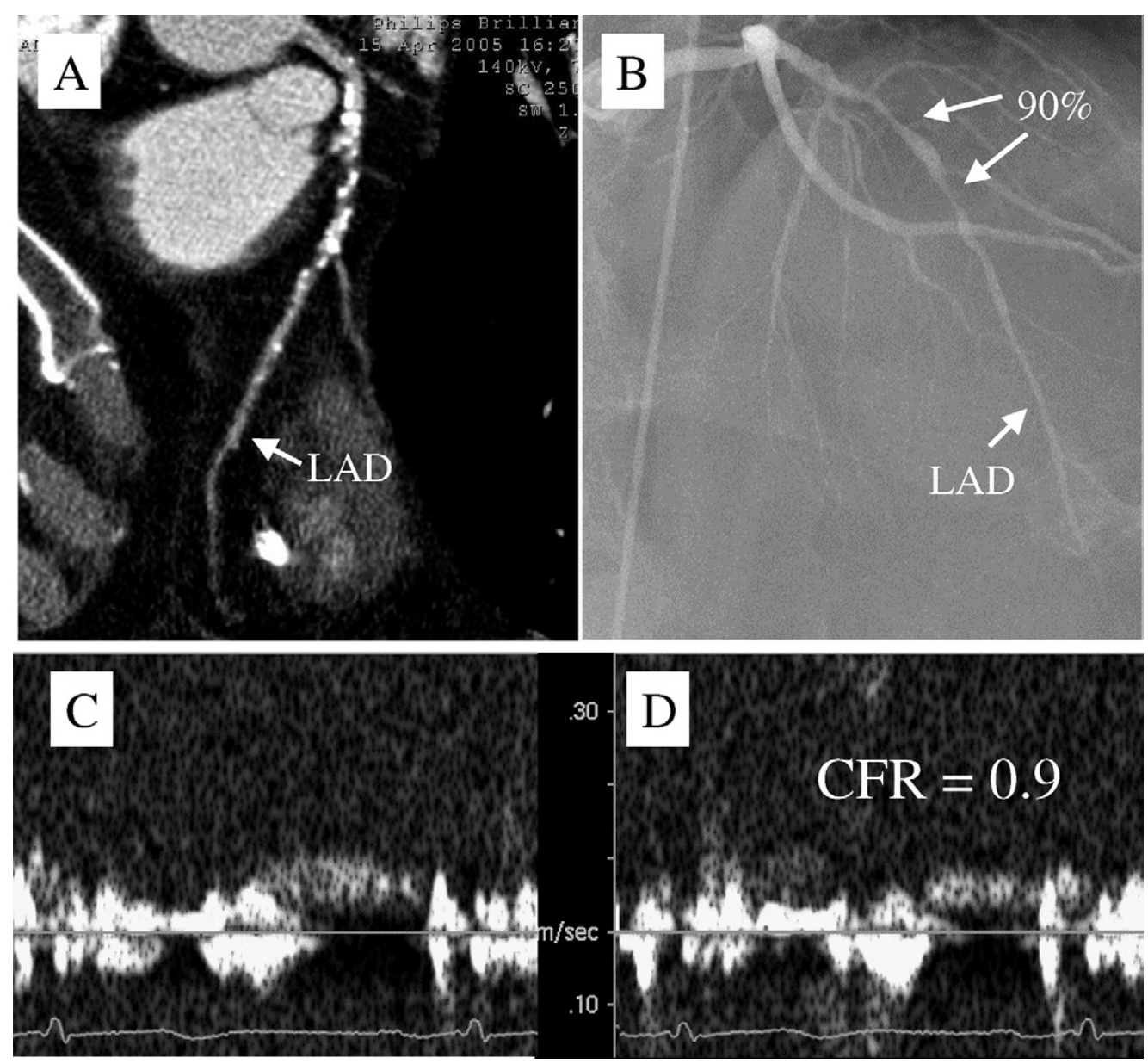

Figure 1. Typical patient with inconclusive multidetector computed tomographic findings (A) because of large amount of calcium in proximal and middle tract of LAD coronary artery. $(B)$ Invasive coronary angiography documented presence of tandem $90 \%$ stenosis at level of proximal and middle LAD coronary segments. $(C, D)$ Transthoracic Doppler ultrasound scan showing impaired noninvasive CFR of 0.9, predicting subocclusion of LAD artery.

ultrasound beam. The LAD flow velocity was measured by pulsed Doppler ultrasonography. In patients with a LIMALAD graft, to prevent the influence of competitive flow on the LIMA, the transthoracic color Doppler echocardiographic assessment of graft function was performed in the distal LAD artery. ${ }^{12}$ We measured the CFR in the distal LAD alone, because it best reflected the global function of the LIMA-to-LAD conduit. When the LAD artery proximal to the anastomosis is totally occluded, or significantly stenotic, it does not affect the distal LAD runoff, which is provided by the LIMA graft. When the proximal LAD artery is not significantly stenotic, its competitive flow can blunt the CFR in the LIMA. In no case does competitive flow affect the CFR in the distal LAD artery. ${ }^{12}$ All the studies were digitally acquired and stored for off-line analysis. The systolic and diastolic peak and mean flow velocities were measured in the LAD artery. Hyperemic flow was measured during 90 seconds of venous adenosine infusion (140 $\mu \mathrm{g} / \mathrm{kg} / \mathrm{min})$, and CFR was calculated as the ratio between the hyperemic and baseline peak flow velocities. For each test, 3 baseline and 3 hyperemic Doppler velocities were computed and averaged. All patients had a continuous heart rate and electrocardiographic monitoring. The blood pressure was recorded at baseline, during the adenosine infusion, and at recovery, for each test. A CFR of $<2$ was used to predict significant $(\geq 70 \%)$ stenosis along the LAD artery. ${ }^{8-12}$ In our laboratory, the inter- and intraobserver variability of coronary Doppler ultrasound measurements was $3.2 \%$ and $2 \%$, respectively, ${ }^{9}$ and the intraindividual variability never exceeded $2 \mathrm{~cm} / \mathrm{s}$, providing a maximal $\pm 6 \%$ difference in relative terms. ${ }^{9}$ The flow parameters were measured blind to the angiographic and multidetector computed tomographic results.

Invasive coronary angiography was the reference diagnostic method and was performed using the percutaneous femoral approach, with a GE Innova 2001 angiographic system (Milwaukee, Wisconsin). The LIMA grafts and native coronary arteries were selectively visualized. The offline maximum percent lumen reduction was determined for each stenotic segment using standard quantitative coronary angiographic software (QCA Medis, Medical Imaging Systems, Leiden, The Netherlands). ${ }^{16}$ The coronary angiographic studies were analyzed independently by 2 on-site cardiologists, who were unaware of the findings from MDCT and CFR. Differences in interpretation were resolved by consensus or by a binding third opinion. Stenosis $\geq 70 \%$ was considered significant. 

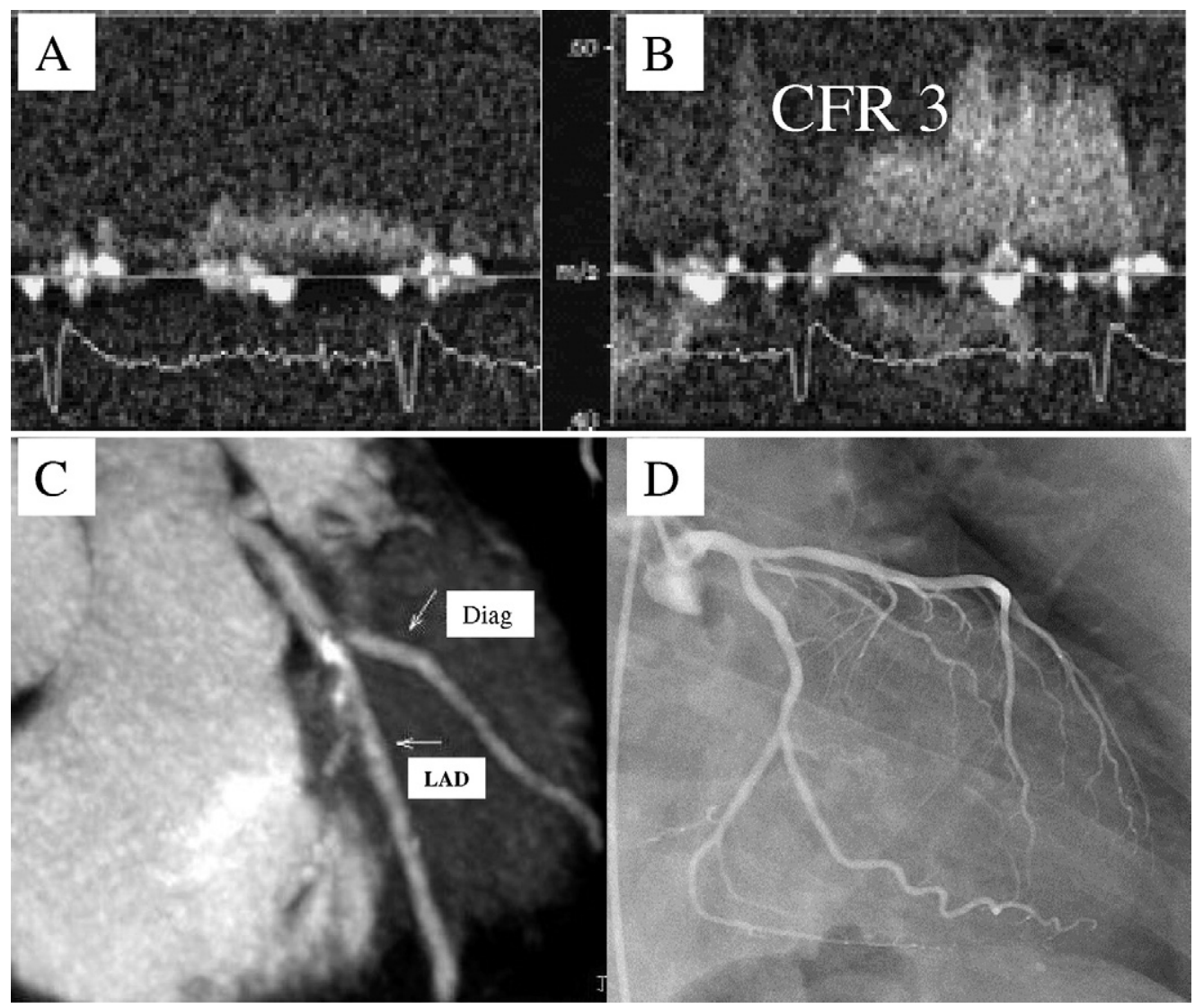

Figure 2. Patient with disagreement between transthoracic Doppler echocardiographic and multidetector computed tomographic findings. (A, $B$ ) Normal CFR measurement (CFR of 3) of LAD by TDE. (C) Multidetector computed tomographic coronary angiography predicted significant calcified stenosis along LAD, close to first diagonal (Diag). (D) Invasive coronary angiography showed mild, not significant, stenosis of proximal LAD.

Data are expressed as the mean $\pm \mathrm{SD}$ or $\mathrm{SE}$ (when the variables were percent data). The BMDP software (University of California Press, Berkeley, California, 1990), release 7.0 (BMDP-Dynamic, BMDP Statistical Software, Cork, Ireland, 1993), was used. Intergroup differences were assessed by $t$ test with separate variance (BMDP-7D). Sensitivity, specificity, and diagnostic accuracy were calculated and compared using chi-square results (BMDP-4F), as reported previously. ${ }^{12}$ The chi-square test was used to test significance for proportions. Correlations were analyzed by linear regression by groups (BMDP-1R), and F statistics were used to assess the significance. The presence (coded 1) or absence (coded 0) of significant $(\geq 70 \%)$ stenosis on quantitative coronary angiography was modeled in all patients in whom MDCT and CFR by TDE were also obtained, using a logistic neural network $(y=1 /[1+\exp (-1 \times$ coefficient)]) fed with a set of covariates, including actual LAD stenosis by MDCT, CFR by TDE, age, heart rate and blood pressure changes, double product and body surface area (all as continuous covariates), smoking habits, hypertension, hypercholesterolemia, gender, and presence of diabetes (all as dichotomous covariates), and number of diseased vessels (as a categorical covariate). For this purpose, Tiberius data mining software, version 5.4.3 (available from: www.tiberius.biz) was used. The software calculated the coefficients and presented the importance of predictive covariates in each model in graph form. The Gini's coeffi- cient is the area between the diagonal and the curve, and the area under curve is the total area under a receiver operating curve. It follows that the area under the curve $=($ Gini $\times$ $0.5)+0.5$. The area under the curve and Gini plots are the same; however, Gini is used in economics and medical statisticians prefer the area under the curve. We used Gini, because this is the metric present in the Tiberius software. A value of $\mathrm{p}<0.05$ was considered statistically significant.

\section{Results}

The LAD artery CFR was measurable in 141 (98\%) of 144 patients, and the LAD artery quantitative coronary angiography by MDCT was measurable in 131 (91\%) of 144 patients $(\mathrm{p}<0.02)$. During the computed tomographic scans, the heart rate was $56 \pm 4$ beats/min. The causes for failed transthoracic Doppler echocardiographic measurements were hyperventilation during adenosine infusion (2 patients) and a poor acoustic window (1 patient). The reasons for failed MDCT included a large amount of coronary calcium (9 patients; Figure 1), blooming from a metallic LAD stent (1 patient), inadequate opacification of the distal LAD despite a well-functioning LIMA graft (1 patient), and unpredictable ventricular ectopic beats (2 patients).

Significant $(\geq 70 \%)$ LAD stenosis was present at invasive angiography in 29 of 144 patients (12 of 59 patients with suspected coronary artery disease and 17 of 85 patients 

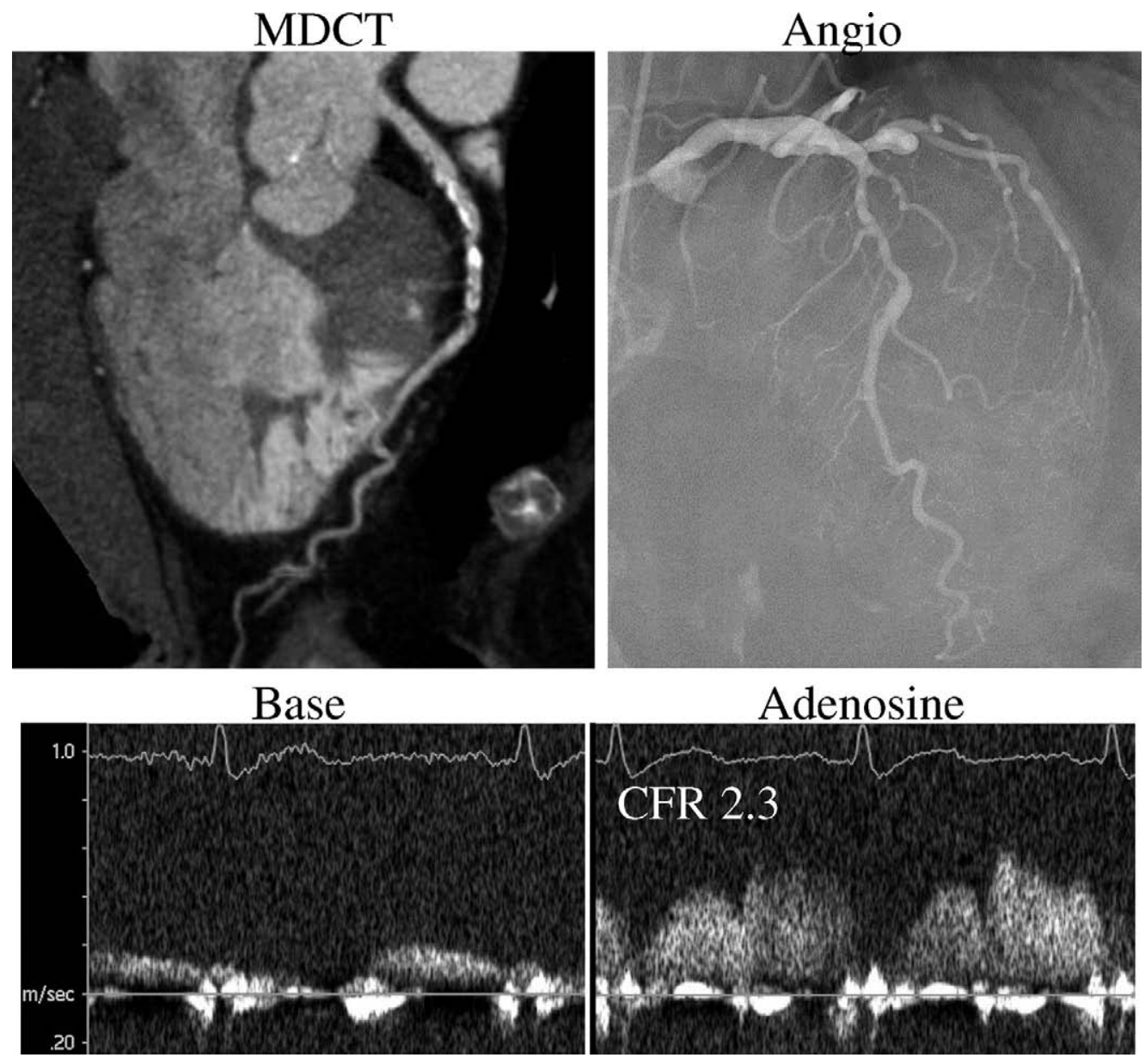

Figure 3. (Upper panels) Noninvasive (MDCT) and invasive angiography. Both techniques identified intermediate LAD stenosis. Measurement of LAD CFR by TDE was 2.3 (lower panels), which agreed with both (noninvasive and invasive) coronary angiographic techniques.

with proven coronary artery disease). None of the patients with a LIMA graft had significant stenosis along the LIMAto-distal LAD conduit on angiography (the LAD proximal to the anastomosis was occluded in 16 patients and significantly stenotic in 4); none of the patients with a LAD stent had significant in-stent restenosis on angiography (stenosis $17 \pm 3 \%$, range $0 \%$ to $40 \%$ ). CFR predicted significant LAD stenosis in 26 of 29 patients and nonsignificant LAD stenosis in 107 of 112 patients. MDCT identified significant LAD stenosis in 20 of 25 patients (in 10 of 12 with suspected coronary artery disease and in 10 of 13 with proven coronary artery disease) and nonsignificant stenosis in 98 of 106 patients. The reasons for missing significant LAD stenosis was poor vessel opacification in 3 patients, motion artifact in 1 , and a small $(<2.5-\mathrm{mm})$ vessel caliper in 1 . Good function of the LIMA-to-LAD conduit was correctly predicted by CFR in 20 of 20 patients and by MDCT in 19 of 20 patients, with 1 failure owing to incomplete visualization of the distal runoff. In the LAD-stented patients, CFR correctly predicted no significant stenosis in all patients, but MDCT had 1 false-positive result and 1 notassessable case because of metallic blooming. Thus, the prediction of significant LAD stenosis was slightly better with TDE ( $\mathrm{n}=141$, sensitivity $90 \%$, specificity $96 \%$, positive predictive value $84 \%$, negative predictive value $97 \%$, diagnostic accuracy $94 \%$, and chi-square $=97.5)$ than with MDCT $(\mathrm{n}=131$, sensitivity $80 \%$, specificity $93 \%$, positive predictive value $71 \%$, negative predictive value $95 \%$, diagnostic accuracy $90 \%$, and chi-square $=63.2$; Figure 2 ), with the difference statistically significant $(\mathrm{p}<0.0001)$. When TDE and MDCT agreed on the prediction of significant LAD stenosis, the results improved $(\mathrm{n}=117$, sensitivity $91 \%$, specificity $97 \%$, positive predictive value $86 \%$, negative predictive value $98 \%$, diagnostic accuracy $96 \%$, and chi-square $=86.1$; Figures 3 and 4 ). A good correlation $\left(\mathrm{r}^{2}=0.69, \mathrm{~F}=287, \mathrm{p}<0.0001\right)$ was found between invasive and noninvasive quantitative coronary angiography, but a slight overestimation of LAD stenosis was seen with MDCT (LAD stenosis by invasive angiography $25.8 \pm$ 2.8\%; LAD stenosis by MDCT $31.8 \pm 2.9 \%$; Figure 5). Of the 13 patients with unfeasible MDCT, 4 had significant LAD stenosis and 9 nonsignificant stenosis. All 13 patients had feasible CFR assessment of the LAD by TDE, with a diagnostic accuracy of $100 \%$.

Among the 128 patients for whom quantitative coronary angiography, MDCT, and CFR as assessed by TDE were obtained, a neural network build ensemble with auto variable removal indicated that of 13 covariates used to predict the presence of $\geq 70 \%$ LAD stenosis at invasive quantitative coronary angiography, $\geq 70 \% \mathrm{LAD}$ stenosis at MDCT, CFR 

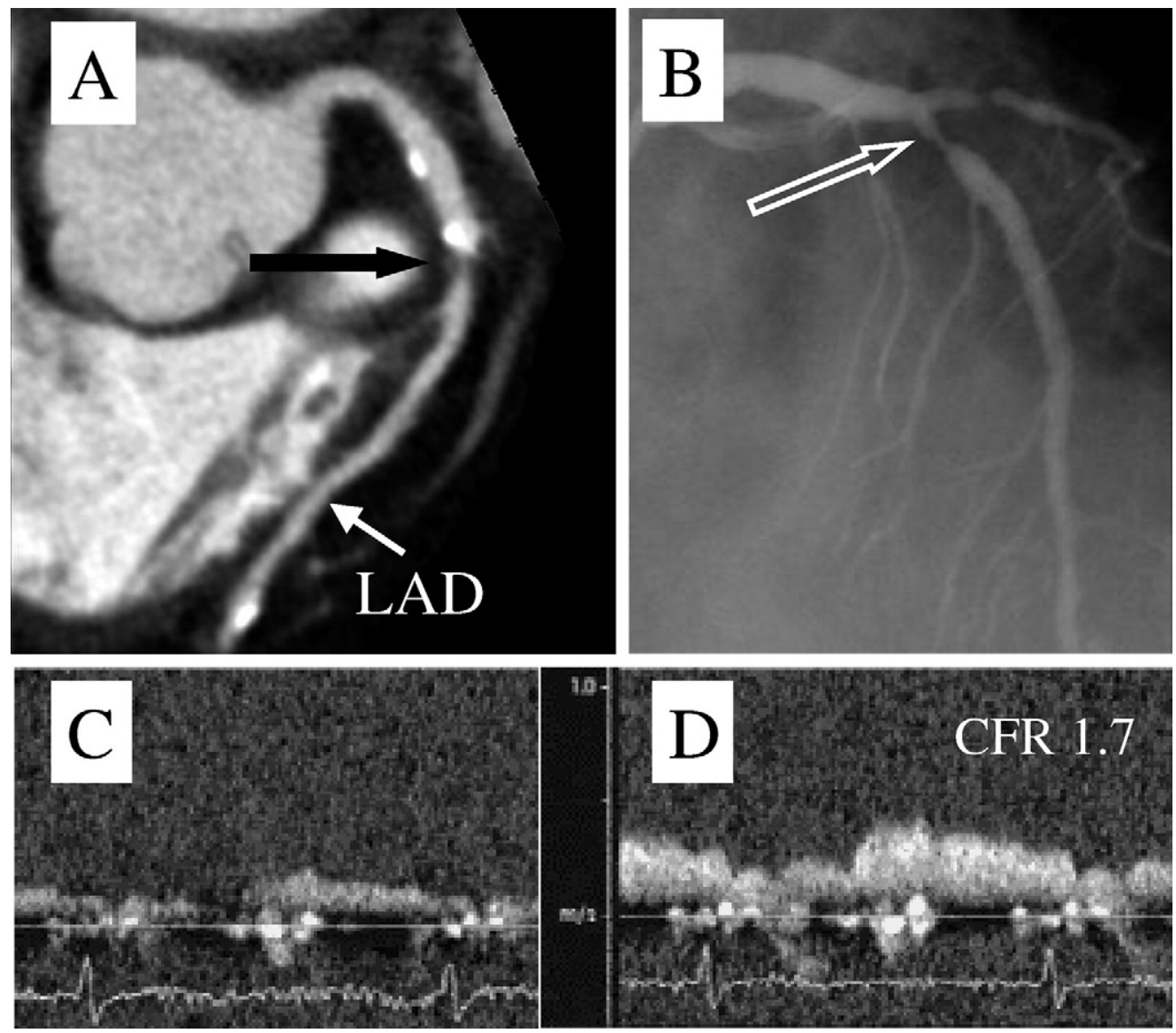

Figure 4. (Upper panels) Noninvasive (MDCT) and invasive coronary angiography, which detect an 80\% middle-tract LAD stenosis (black and empty white arrows). LAD CFR by TDE was 1.7 (lower panels), confirming coronary angiographic findings.

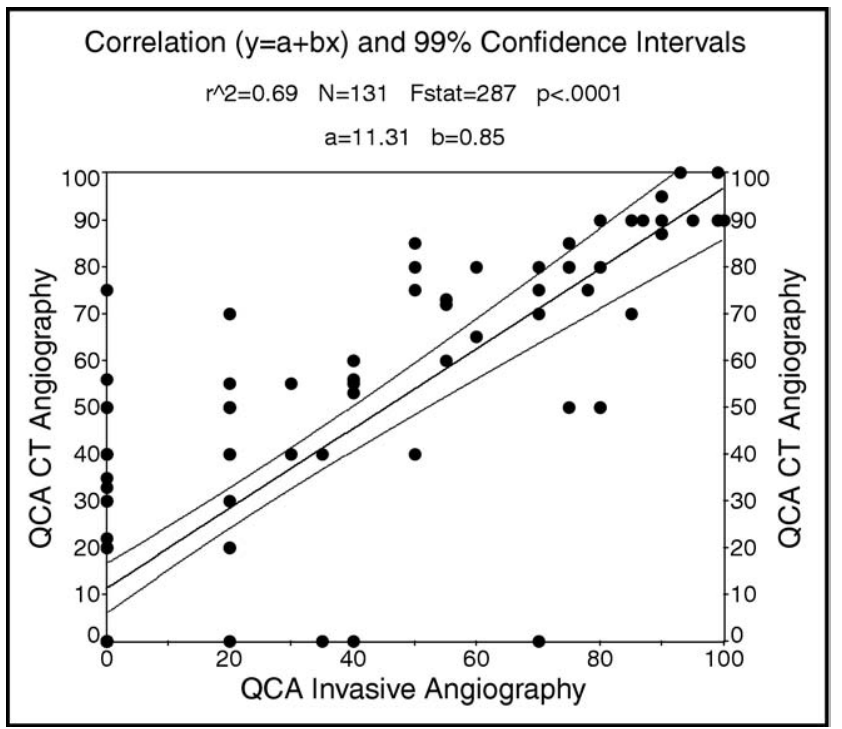

Figure 5. Linear correlation (and 99\% confidence intervals) between quantitative coronary angiography (QCA) as obtained by invasive angiography ( $\mathrm{x}$ axis) and multidetector computed tomography (CT angiography, y axis) in detecting LAD coronary artery stenosis in 131 patients. Parameters $a$ and $b$ of regression equation shown to enable easy calculation of LAD stenosis prediction, which was slightly overestimated if invasive angiography considered the standard. These methods were highly significantly correlated and $69 \%$ of the variance was explained by this correlation. by TDE, smoking habit, hypertension, baseline rate pressure product, age, and gender contributed (the latter 5 modestly, however) with a Gini model average of 0.97 (Figure 6). With the neural network, the average model Gini when the input was randomized 3 times, gave the following results: 0.11 for CFR by TDE (variable 1), 0.97 for age (variable 2), and 0.97 for female gender (variable 3), and the remaining covariates were removed. The final calculated coefficient for the neural network model was a coefficient of $-2.58+$ $(\tanh [-3.19+($ variable $1 \times-7.88)+($ variable $2 \times 0.81)+$ $($ variable $3 \times-1.46)] \times 1,95)+(\tanh [-1.86+($ variable $1 \times-4.48)+($ variable $2 \times 0.51)+($ variable $3 \times-0.79)] \times$ 0.75 ); where coefficient $=$ coefficient $\times 2.01$; and probability of $>70 \%$ LAD stenosis at quantitative coronary angiography $=1 /(1+\exp [-1 \times$ coefficient $])$. The area under the curve for the neural network model was 0.99 (Gini 0.98 and residual mean squared [RMS] error 0.18).

\section{Discussion}

Selective coronary angiography is the reference standard technique for evaluating coronary artery disease, but it cannot be routinely or serially used to assess modifications in vessel anatomy and flow. MDCT has been suggested as an alternative noninvasive tool, providing good results in patients with coronary grafts and stents. ${ }^{17-19}$ However, some technical limitations and the radiation exposure prevents its 


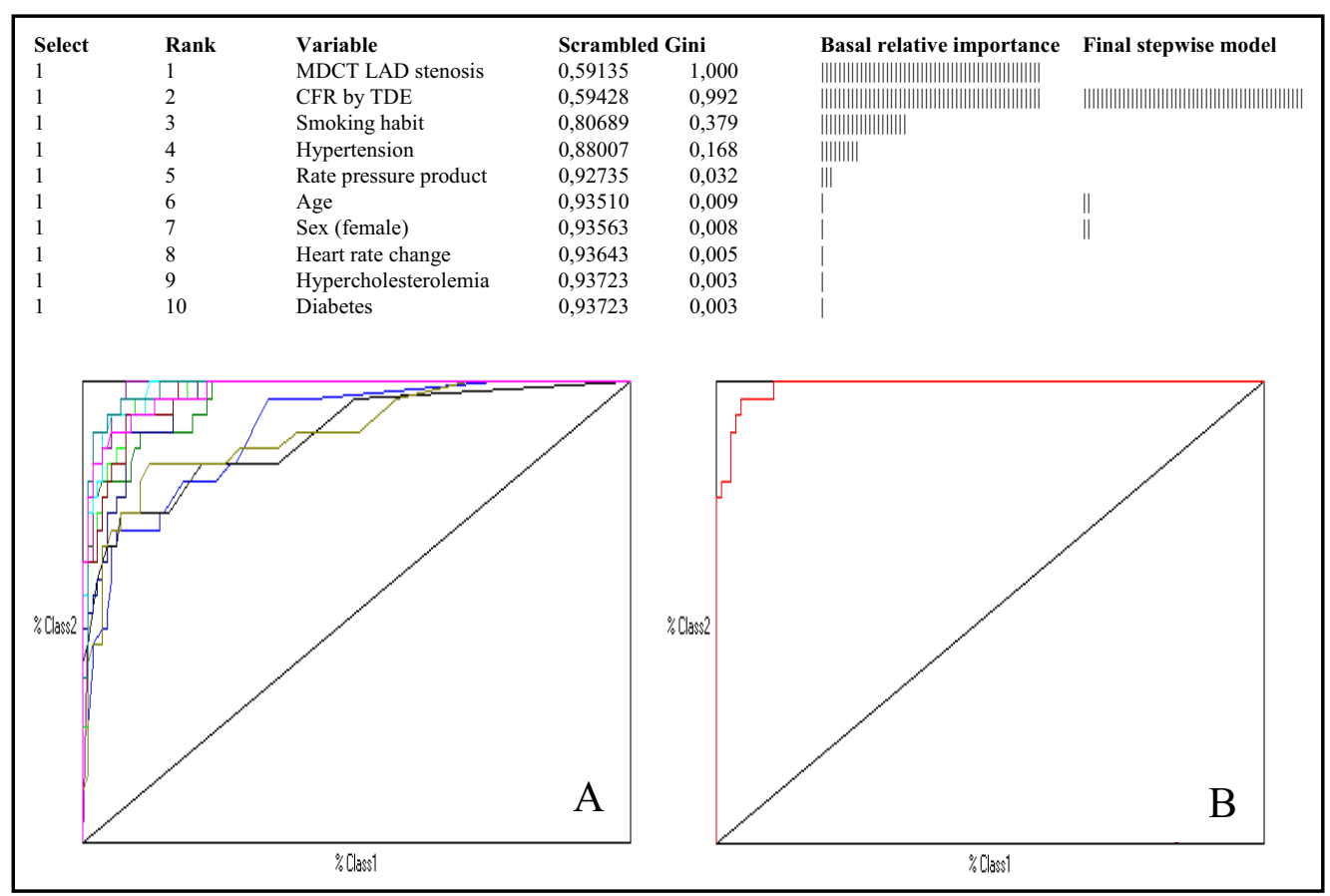

Figure 6. Prediction of significant ( $\geq 70 \%$ ) LAD stenosis at coronary angiography by neural network in which basal situation and validation of 128 patients in whom both quantitative coronary angiography (QCA) and MDCT were obtained, along with CFR, as assessed by TDE. A neural network build ensemble with auto variable removal (by Tiberius software, available from: www.tiberius.biz) indicated that of 13 covariates used to predict significant LAD stenosis at invasive QCA, significant LAD stenosis at MDCT, CFR by TDE, smoking habit, hypertension, baseline rate pressure product, age, and gender contributed, with Gini model average of 0.97 . With stepwise neural network model, graphed-synthetic relative importance shows that most important covariate was CFR by TDE, followed by age and female gender, with remaining covariates removed. (A, $B$ ) Receiver operating characteristic area under curve (ROC AUC) for neural network build ensemble and stepwise model, respectively. Stepwise neural network model had a Gini coefficient of 0.98 (ROC AUC $=0.99$ ).

widespread use. Although these methods were significantly correlated in the present investigation, with $69 \%$ of the variance explained by this correlation (Figure 5), LAD stenosis was slightly overestimated by MDCT, in accordance with the published data. ${ }^{1,3,20,21}$ The limited spatial and temporal resolution of MDCT compared to invasive coronary angiography leads to image degradation by motion and calcium. The bright, blooming signal from high-density objects such as calcified plaques or metallic stents magnifies their true size, leading to an overestimation of the stenosis severity. This limitation was underlined in a recent report that showed that the amount of calcium significantly reduced the specificity of MDCT to discriminate significant $(50 \%)$ stenosis (diagnostic accuracy $86 \%$ in patients with a coronary artery calcium score of $\leq 400 \%$ vs $53 \%$ in those with $\geq 400$ Agatston units; $p \leq 0.0003$ ). ${ }^{21}$ Finally, unpredictable ventricular ectopic beats limit the feasibility of MDCT, decreasing the number of patients that can be effectively studied and/or correctly analyzed.

Our results have shown that the adjunct of CFR improves the diagnostic accuracy of MDCT when the prediction of significant LAD stenosis is the aim, a finding that was particularly stressed by the logistic neural network analysis among the 128 patients who had undergone all techniques explored in the present study (Figure 6). Because the area under the receiver operating characteristic curve was almost 1 , it can be concluded that the prediction of significant LAD stenosis was excellent. This reinforces the accuracy results obtained in subgroups of patients in whom either the bivariate assessment with CFR only $(n=141)$ or LAD stenosis by MDCT only $(\mathrm{n}=131)$ or the concordant results $(\mathrm{n}=$ 117) using both 2 methods were obtained.

Functional information on the physiologic significance of coronary lesions is important for clinical decision-making related to revascularization and prognosis. It appears reasonably safe to defer percutaneous coronary intervention in patients with intermediate coronary lesions (50\% to 69\%) if the CFR is $\geq 2 .^{22}$ Moreover, patients with intermediate coronary stenoses who present with a good fractional flow reserve $(\geq 0.75)$ have a low risk of cardiac death or myocardial infarction ( $<1 \%$ annually), a risk that is not decreased by stenting the related lesion. ${ }^{23}$

Simultaneous morphologic and functional (metabolic and/or perfusion) information obtained from hybrid positron emission computed tomography and single photon emission computed tomography-computed tomography scans in a single test has been suggested. ${ }^{7}$ However, hybrid scanners present problems with image acquisition, radiation exposure, and cost.

Our results have shown that information on the physiologic effect of LAD stenosis can be noninvasively obtained using ultrasonography, which might overcome some of the limitations of computed tomographic angiography, 1,3,20,21 mostly when doubt exists about the estimation of the coronary artery stenosis severity or artifacts are present. A CFR cutoff of $<2.0$ was more accurate than MDCT for the detection of significant $(\geq 70 \%)$ LAD stenosis on angiography. When the 2 noninvasive techniques were concordant, the diagnostic accuracy was very high (95.7\%).

Finally, the feasibility of CFR in the LAD artery was greater than that of MDCT (98\% vs $91 \%$, respectively, 
$\mathrm{p}<0.02)$. Remarkably, when MDCT was not interpretable (about 10\% of the cases) TDE allowed a diagnostic accuracy of $100 \%$.

Our study was undertaken with a 40-slice scanner, which has a slightly lower resolution power than the more powerful 64-slice scanners used in recent clinical practice. $^{1,3,20,21}$ However, the limitations encountered in our study were similar to those reported with 64-slice MDCT, as well as with the results of coronary stenosis quantification, in either single-center ${ }^{1,3,4}$ or multicenter studies. ${ }^{20,21}$ The most important limitation of TDE is that only the LAD artery can be easily studied, and other arteries can be studied in only $50 \%$ to $80 \%$ of the patients. ${ }^{24,25}$ The other potential limitation of TDE is the influence of microcirculation on CFR. ${ }^{26}$ However, in our experience with adenosine (endothelial independent) vasodilation, the bias introduced by microcirculatory abnormality has been negligible. ${ }^{27,28}$

1. Hoffmann MH, Shi H, Schmitz BL, Schmid FT, Lieberknecht M, Schulze R, Ludwig B, Kroschel U, Jahnke N, Haerer W, Brambs HJ, Aschoff AJ. Noninvasive coronary angiography with multislice computed tomography. JAMA 2005;293:2471-2478.

2. Leschka S, Alkadhi H, Plass A, Desbiolles L, Grünenfelder J, Marincek $\mathrm{B}$, Wildermuth S. Accuracy of MSCT coronary angiography with 64-slice technology: first experience. Eur Heart $J$ 2005;26:1482-1487.

3. Raff GL, Gallagher MJ, O’Neill WW, Goldstein JA. Diagnostic accuracy of noninvasive coronary angiography using 64-slice spiral computed tomography. J Am Coll Cardiol 2005;46:552-557.

4. Leber AW, Knez A, von Ziegler F, Becker A, Nikolaou K, Paul S, Wintersperger B, Reiser M, Becker CR, Steinbeck G, Boekstegers P. Quantification of obstructive and nonobstructive coronary lesions by 64-slice computed tomography: a comparative study with quantitative coronary angiography and intravascular ultrasound. J Am Coll Cardiol 2005;46:147-154.

5. Mollet NR, Cademartiri F, van Mieghem CA, Runza G, McFadden EP, Baks T, Serruys PW, Krestin GP, de Feyter PJ. High-resolution spiral computed tomography coronary angiography in patients referred for diagnostic conventional coronary angiography. Circulation 2005;112: $2318-2323$.

6. Fine JJ, Hopkins CB, Ruff N, Newton FC. Comparison of accuracy of 64-slice cardiovascular computed tomography with coronary angiography in patients with suspected coronary artery disease. Am J Cardiol 2006;97:173-174.

7. Di Carli MF, Hachamovitch R. New technology for noninvasive evaluation of coronary artery disease. Circulation 2007;115:1464-1480.

8. Hozumi T, Yoshida K, Ogata Y, Akasaka T, Asami Y, Takagi T, Morioka S. Noninvasive assessment of significant left anterior descending coronary artery stenosis by coronary flow velocity reserve with transthoracic color Doppler echocardiography. Circulation 1998; 97:1557-1562.

9. Pizzuto F, Voci P, Mariano E, Puddu PE, Sardella G, Nigri A. Assessment of flow velocity reserve by transthoracic Doppler echocardiography and venous adenosine infusion before and after left anterior descending coronary stenting. J Am Coll Cardiol 2001;38:155-162.

10. Ruscazio M, Montisci R, Colonna P, Caiati C, Chen L, Lai G, Cadeddu $\mathrm{M}$, Pirisi R, Iliceto S. Detection of coronary restenosis after coronary angioplasty by contrast-enhanced transthoracic echocardiographic Doppler assessment of coronary flow velocity reserve. J Am Coll Cardiol 2002;40:896-903.

11. Pizzuto F, Voci P, Mariano E, Puddu PE, Chiavari PA, Romeo F. Noninvasive coronary flow reserve assessed by transthoracic coronary Doppler ultrasound in patients with left anterior descending coronary artery stents. Am J Cardiol 2003;91:522-526.

12. Pizzuto F, Voci P, Mariano E, Puddu PE, Aprile A, Romeo F. Evaluation of flow in the left anterior descending coronary artery but not in the left internal mammary artery graft predicts significant stenosis of the arterial conduit. J Am Coll Cardiol 2005;45:424-432.
13. Pizzuto F, Voci P, Puddu PE, Chiricolo G, Borzi M, Romeo F. Functional assessment of the collateral-dependent circulation in chronic total coronary occlusion using transthoracic Doppler ultrasound and venous adenosine infusion. Am J Cardiol 2006;98:197-203.

14. Manzke R, Grass M, Nielsen T, Shechter G, Hawkes D. Adaptive temporal resolution optimization in helical cardiac cone beam CT reconstruction. Med Phys 2003;30:3072-3080.

15. Vembar M, Garcia MJ, Heuscher DJ, Haberl R, Matthews D, Bohme GE, Greenberg NL. A dynamic approach to identifying desired physiological phases for cardiac imaging using multislice spiral CT. Med Phys 2003;30:1683-1693.

16. Tuinenburg JC, Koning G, Hekking E, Desjardins C, Harel F, Bilodeau L, van Weert AW, Lespérance J, Reiber JH. One core laboratory at two international sites, is that feasible? An inter-core laboratory and intraobserver variability study. Cath Cardiovasc Interv 2002;56:333-340.

17. Martuscelli E, Romagnoli A, D’Eliseo A, Tomassini M, Razzini C, Sperandio M, Simonetti G, Romeo F, Mehta JL. Evaluation of venous and arterial conduit patency by 16-slice spiral computed tomography. Circulation 2004;110:3234-3238.

18. Ropers D, Pohle FK, Kuettner A, Pflederer T, Anders K, Daniel WG, Bautz W, Baum U, Achenbach S. Diagnostic accuracy of noninvasive coronary angiography in patients after bypass surgery using 64-slice spiral computed tomography with $330-\mathrm{ms}$ gantry rotation. Circulation 2006;114:2334-2341.

19. Cademartiri F, Schuijf JD, Pugliese F, Mollet NR, Jukema JW, Maffei E, Kroft LJ, Palumbo A, Ardissino D, Serruys PW, Krestin GP, Van der Wall EE, de Feyter PJ, Bax JJ. Usefulness of 64-slice multislice computed tomography coronary angiography to assess in-stent restenosis. J Am Coll Cardiol 2007;49:2204-2210.

20. Budoff MJ, Dowe D, Jollis JG, Gitter M, Sutherland J, Halamert E, Scherer M, Bellinger R, Martin A, Benton R, Delago A, Min JK. Diagnostic performance of 64-multidetector row coronary computed tomographic angiography for evaluation of coronary artery stenosis in individuals without known coronary artery disease: results from the prospective multicenter ACCURACY (Assessment by Coronary Computed Tomographic Angiography of Individuals Undergoing Invasive Coronary Angiography) trial. J Am Coll Cardiol 2008;52:1724-1732.

21. Miller JM, Rochitte CE, Dewey M, Arbab-Zadeh A, Niinuma H, Gottlieb I, Paul N, Clouse ME, Shapiro EP, Hoe J, Lardo AC, Bush DE, de Roos A, Cox C, Brinker J, Lima JAC. Diagnostic performance of coronary angiography by 64-row CT. N Engl J Med 2008;359: 2324-2336.

22. Ferrari M, Schnell B, Werner GS, Figulla HR. Safety of deferring angioplasty in patients with normal coronary flow velocity reserve. J Am Coll Cardiol 1999;33:82-87.

23. Pijls NH, van Schaardenburgh P, Manoharan G, Boersma E, Bech JW, Van't Veer M, Bär F, Hoorntje J, Koolen J, Wijns W, de Bruyne B. Percutaneous coronary intervention of functionally nonsignificant stenosis: 5-year follow-up of the DEFER study. J Am Coll Cardiol 2007;49:2105-2111.

24. Voci P, Pizzuto F, Mariano E, Puddu PE, Chiavari P, Romeo F. Measurement of coronary flow reserve in the anterior and posterior descending coronary arteries by transthoracic Doppler ultrasound. Am J Cardiol 2002;90:988-991.

25. Lethen H, Tries HP, Kersting S, Lambertz H. Validation of noninvasive assessment of coronary flow velocity reserve in the right coronary artery. A comparison of transthoracic echocardiographic results with intracoronary Doppler flow wire measurements. Eur Heart J 2003;24: $1567-1575$.

26. Galderisi M. Diastolic dysfunction and diabetic cardiomyopathy: evaluation by Doppler echocardiography. J Am Coll Cardiol 2006;48: $1548-1551$.

27. Pizzuto F, Voci P, Mariano E, Puddu PE, Spedicato P, Romeo F. Coronary flow reserve of the angiographically normal left anterior descending coronary artery in patients with remote coronary artery disease. Am J Cardiol 2004;94:577-582.

28. Citro R, Voci P, Pizzuto F, Maione AG, Patella MM, Bossone E, Provenza G, Gregorio G, Mariano E, Feinstein M, Athanassopoulos G, Puddu PE. Clinical value of echocardiographic assessment of coronary flow reserve after left anterior descending coronary artery stenting in an unselected population. J Cardiovasc Med 2008;9:1254-1259. 